\title{
Home paint exposures and risk of childhood acute lymphoblastic leukemia: Findings from the Childhood Leukemia International Consortium
}

\author{
Helen D Bailey ${ }^{1}$, Catherine Metayer ${ }^{2}$, Elizabeth Milne ${ }^{3}$, Eleni Petridou ${ }^{4}$, Claire Infante- \\ Rivard $^{5}$, Logan G Spector ${ }^{6}$, Jacqueline Clavel ${ }^{7}$, John D Dockerty ${ }^{8}$, Luoping Zhang ${ }^{2}$, Bruce \\ K Armstrong ${ }^{9,10}$, Jérémie Rudant ${ }^{7}$, Lin Fritschi ${ }^{11}$, Alicia Amigou ${ }^{7}$, Emmanouel \\ Hatzipantelis $^{12}$, Alice Y Kang ${ }^{2}$, Eftychia Stiakaki ${ }^{13}$, and Joachim Schüz ${ }^{1}$ \\ ${ }^{1}$ International Agency for Research on Cancer (IARC), Section of Environment and Radiation, \\ Lyon, France ${ }^{2}$ University of California, Berkeley, School of Public Health, Berkeley, United States \\ ${ }^{3}$ Telethon Kids Institute, University of Western Australia, Perth Australia ${ }^{4}$ Department of Hygiene, \\ Epidemiology and Medical Statistics, Medical School, National and Kapodistrian University of \\ Athens, Medical School, Athens, Greece ${ }^{5}$ Department of Epidemiology, Biostatistics, and \\ Occupational Health, Faculty of Medicine, McGill University, Canada ${ }^{6}$ Division of Epidemiology \\ Clinical Research, Department of Pediatrics and Masonic Cancer Center, University of \\ Minnesota, Minneapolis, United States ${ }^{7}$ Inserm U1153, Epidemiology and Biostatistics Sorbonne \\ Paris Cité Center (CRESS), Epidemiology of childhood and adolescent cancers team (EPICEA), \\ Paris-Descartes University, Villejuif, France ${ }^{8}$ Dean's Department and Department of Preventive \\ and Social Medicine, Dunedin School of Medicine, University of Otago, Dunedin, New Zealand \\ ${ }^{9}$ University of Sydney, Sydney School of Public Health, New South Wales, Australia ${ }^{10}$ Sax \\ Institute, Ultimo, NSW, Australia ${ }^{11}$ Curtin University, School of Public Health, Perth, Australia \\ ${ }^{12}$ Pediatric Hematology Oncology Unit, 2nd Pediatric Department of Aristotle University, AHEPA \\ General Hospital, Thessaloniki, Greece ${ }^{13}$ Department of Pediatric Hematology-Oncology, \\ University Hospital of Heraklion, Heraklion, Greece
}

\section{Abstract}

Purpose-It has been suggested that home paint exposure increases the risk of childhood acute lymphoblastic leukemia (ALL).

\begin{abstract}
Methods-We obtained individual level data from eight case-control studies participating in the Childhood Leukemia International Consortium. All studies had home paint exposure data (sometimes including lacquers and varnishes) for the pregnancy period with additional data for the 1-3 month period before conception in five, the year before conception in two, and the period after birth in four studies respectively. Cytogenetic subtype data were available for some studies. Data
\end{abstract}

\footnotetext{
Address for correspondence: Helen Bailey, Section of Environment and Radiation, International Agency for Research on Cancer, 150 cours Albert Thomas, Lyon 69372 Cedex 08, France. Telephone: [+33] (0)4 7273 8310. Fax: [+33] (0)4 72 73 8320 baileyh@fellows.iarc.fr.

Authorship: All authors are principal investigators, co-investigators or designated collaborators of participating CLIC studies

The authors declare that they have no conflict of interest.
} 
were harmonized to a compatible format. Pooled analyses of individual data were undertaken using unconditional logistic regression.

Results-Based on 3,002 cases and 3,836 controls, the pooled odds ratio (OR) for home paint exposure in the 1-3 months before conception and risk of ALL was 1.54 (95\% confidence interval (CI) 1.28, 1.85), while based on 1160 cases and 1641 controls for exposure in the year before conception it was 1.00 (95\% CI 0.86, 1.17). For exposure during pregnancy, using 4,382 cases and 5,747 controls, the pooled OR was $1.14(95 \%$ CI 1.04, 1.25) and for exposure after birth, the OR was 1.22 (95\% CI 1.07, 1.39), based on data from 1,962 cases and 2,973 controls. The risk was greater for certain cytogenetic subtypes and if someone other than the parents did the painting.

Conclusions-Home paint exposure shortly before conception, during pregnancy and/or after birth appeared to increase the risk of childhood ALL. It may be prudent to limit exposure during these periods.

\section{Keywords}

paint; acute lymphoblastic leukemia; childhood; pooled analysis

\section{Introduction}

Acute lymphoblastic leukemia (ALL), the most common childhood malignancy, occurs mainly in children under five years of age, suggesting a role for parental exposures before birth or the child's exposure in early childhood. Exposure to house paints has been suggested to be one potentially hazardous exposure in this period (1). ALL is a relatively rare disease in developed countries, with an annual incidence rate of 30-50 per million; hence individual studies rarely have sufficient statistical power to detect an effect, especially when investigating potential risk factors by sub-types that may have differing etiologies. To overcome this problem, we pooled data from studies participating in the Childhood Leukemia International Consortium (CLIC), a multi-national collaboration of case-control studies of childhood leukemia (2). The focus of these analyses was to investigate home paint exposure in relation to ALL. We have previously published findings of pooled analyses investigating parental occupational exposure to paints and the risk of childhood ALL using data from CLIC studies and found no association with paternal occupational exposure around conception, but had insufficient statistical power to investigate maternal occupational exposure during pregnancy (3).

Home paint exposure has been identified as a potential risk factor for ALL in previous studies (4-7), two of which are part of the current pooled analyses $(6,4)$. There is also some evidence of a trend of increasing risk with more rooms painted $(6,7)$ or with painting done by someone other than the parents $(6,4)$, such as a professional painter. In addition, the level of risk may vary by cytogenetic subtype, such as ALL with the ETV6-RunX-1 t12;21 translocation (6,4), the most common subtype of childhood ALL, which may be of prenatal origin (8). A working group of the of the International Agency for Cancer (IARC) Monograph Program on the evaluation of carcinogenic risks to humans concluded in 2010 that there was 'limited evidence that paint exposure is related to childhood leukemia', based mainly on reports of maternal exposure (1). Paint, which is a coating, is a generic name for a 
diverse range of products which in some studies has been broadened to include other coating products such as lacquers and varnishes. All of these contain a large number of individual chemical compounds such as solvents, resins, binders, extenders and pigments, and some of these individual compounds have been classified as human carcinogens or probable or possible human carcinogens such as ethyl acrylate, titanium dioxide and other pigments (1). In the home, paint exposure can occur by actively using paint or spending time in an environment where paint has recently been used.

The aim of these analyses was to investigate whether home paint exposure in the time leading up to conception, during pregnancy or after the child's birth increased the risk of childhood ALL. We also investigated whether the relationship varied by immunophenotype or cytogenetic subtype of ALL.

\section{Methods}

We included data from eight CLIC studies conducted in North America, Europe and Australasia over a 30 year period that had relevant data (Table 1), three of which have previously published findings in relation to home paint exposure $(9,6,4)$. Original data were requested from each study including demographics, disease subtypes, covariates, variables used for control selection or matching factors and any data related to home paint exposure. A summary of study design and participant details, including inclusion criteria, has already been published (2). All studies recruited children under the age of 15 years and were approved by the relevant institutional or regional ethics committees.

\section{Exposure assessment}

We included in the analyses any CLIC study that had a measure of home paint exposure in any of three time periods: before the child's conception, during the pregnancy and after the child's birth. The measures of home paint exposure in each included study are summarized in Table 1. All studies had exposure data for the pregnancy period. Seven studies had exposure data for a period before conception: three had data for exposure in the month before conception (Greece: NARECHEM 1993-1994 and 1996-97; COG-E15); two for the three months before conception (NCCLS and New Zealand) and two for the year before conception (Australia, Canada). Given these differences, we analyzed exposure 1-3 months before conception separately from exposure in the year before conception. Four studies had data for exposure after birth: Australia, Canada and New Zealand, had data for exposure after the child's birth until the reference date, which was the date of diagnosis for the cases and the date of recruitment or questionnaire return for the controls, while the NCCLS had data for exposure until the child's third birthday.

Exposure was defined as 'paint' in one study (France), 'paint or varnish' in two, (Greece: NARECHEM 1993-1994 and 1996-97), 'paints, stains or lacquers' in two studies (NCCLS and COG-E15), paints, lacquers, paint removers, turpentine products or thinners in another (New Zealand) and 'house painting' in two studies (Australia and Canada) (Table 1).

Exposure was considered relevant in either parent before conception, the mother during pregnancy and the child after birth. For the studies with information on household paint use 
in a specified time period (Australia, Canada and NCCLS), we assumed everyone living in the house was exposed. For the other studies, we used the relevant person(s)'s exposure data. The New Zealand study had defined exposure based on maternal exposure in the home or workplace, but as we had previously found that maternal occupational exposure to paints was rare in the other CLIC studies, we used this as a proxy for home exposure (3). We also conducted subgroup analyses in subsets of studies for: the main types of paint used ('not oilbased' or 'oil-based') (Australia, Canada and France); and the trimester of exposure during pregnancy (Australia, Canada and NCCLS); and the person who had done the painting (categorized as 'mother', 'father', 'someone other than the parents') (Australia, Canada and NCCLS). This final group of analyses were only conducted in time periods where the exposure from the parents actively using paint could be more important than spending time in an environment where paint had been used, that is, before conception for both parents and during pregnancy for the mother.

\section{Immunophenotype and cytogenetic classification of ALL}

Information about lineage (B cell and $\mathrm{T}$ cell) was available for all studies. In addition, for B cell ALL cases, data for low hyperdiploidy (47-50 chromosomes) and high hyperdiploidy (51 or more chromosomes) which had been determined using conventional banding karyotypes or fluorescence in situ hybridization screening (FISH) were available for three studies (Australia, France, and NCCLS). For two studies (Australia and NCCLS) data were available for ETV6-RunX-1 gene fusion (cryptic t(12;21) translocations) in B cell ALL cases, determined by FISH or molecular detection of fusion transcripts and for 11q23/MLL rearrangement including either conventional cytogenetic identifying chromosome translocation involving the 11q23 region or $M L L$ gene rearrangement by RT-PCR ( $A F 4$ / $M L L)$ or FISH- $M L L$ break apart. Less common cytogenetic types were not included in our pooled analyses. The number of metaphases was not available in all studies, meaning that the karyotypes with no structural or numerical changes could not be considered normal karyotypes.

All studies routinely extracted existing data from medical records at the time of the diagnosis for all cases. In addition, NCCLS had performed specific analyses at a central laboratory from samples taken at the time of enrollment in the study. Before pooling the cytogenetic data, JC and experts in molecular biology (LZ, MPO) checked the consistency of CLIC data by conducting sex- and age-frequency analyses. In particular, there was no substantial heterogeneity between studies for the B cell cytogenetic abnormalities of interest (low hyperdiploidy, high hyperdiploidy, presence of ETV6-Runx I) or the presence of 11q23/MLL rearrangement, despite the assumed variations in methods across studies and time periods, and the prevalence of these cytogenetic abnormalities matched known distributions from clinical series.

\section{Statistical analyses}

Two distinct analytic approaches were taken. Firstly, study specific odds ratios (ORs) of exposure to paints around the home and risk of ALL were estimated and included in metaanalyses so we could explore heterogeneity between studies. Secondly, individual data were pooled in a single dataset and the pooled ORs estimated. As the findings using both methods 
were similar, the Methods and Results of the meta-analytical approach are presented as Supporting Material.

\section{Pooled analyses}

Unconditional logistic regression (SAS version 9.2, SAS Institute Inc, Cary, NC, USA) was used to estimate pooled ORs and 95\% CIs for paint exposures around the home for the following four time periods: in the 1-3 months before conception, in the year before conception, during pregnancy and between the child's birth and reference date. All models included the child's age, sex, year of birth (grouped into four approximately equal time periods) and ethnicity (Caucasian, European or White versus the rest) and a variable denoting the study of origin. The following variables were considered a priori to be potential confounders and were tested to determine whether they met the empirical definition of confounding; that is, were independently associated with both the exposure and outcome: birth order; birth weight (where available); mother's age and highest education of either parent (secondary education not completed, completed secondary education, and tertiary education); and study-specific matching variables (by allocating all the other studies the same dummy value for each variable). Of these, only highest education of either parent was retained. Subgroup analyses were undertaken for immunophenotypes. We stratified analyses by child's sex, age at diagnosis (0-1 years, 2-4 years, 5-9 years and 10 or more years) and year of birth (before 1996 or later) as there were changes to the maximum levels of volatile organic compounds (VOCs) allowed in paints in the mid 1990's (10,11), and tests for interaction were performed. The analyses for exposures after birth were first run using all studies with data for any time period after birth and then rerun, restricting them to the three studies with exposures up until the reference date. Where there were two or more studies with at least 30 cases with compatible data, sub group analyses were also done by trimester of pregnancy, the person who had done the painting, the type of paint used and the cytogenetic subtype.

To assess whether risk varied between 'before' and 'during' pregnancy, logistic regression models were also repeated using a four level exposure variable: (1) no exposure before or during pregnancy, (2) exposure only before pregnancy, (3) exposure only during pregnancy and (4) exposure during both these time periods. In addition, the OR for exposure in the 1-3 months before or during pregnancy was calculated to allow comparison with previous studies $(7,12)$.

As children with Down syndrome have higher rates of ALL than other children, analyses were repeated excluding these children. Analyses were also repeated adjusting for paternal occupational paint exposure around conception and maternal occupational paint exposure during pregnancy and using combined home and/or occupational paint exposure variables.

\section{Results}

Data were available for up to 4,495 cases and 5,863 controls, depending on the analyses. The demographic characteristics of the pooled sample are shown in Table 2 and those for individual studies in Supplementary Table 2. Cases and controls were generally similar, but control parents were more likely to have had a tertiary education than case parents $(51.0 \%$ vs 
45.6\%). As expected, case children were more likely to have Down Syndrome than control children. Data for exposure during pregnancy were available for all studies and over $97 \%$ of cases and controls, while exposure data for other time periods were available for subsets of studies.

\section{Pooled analyses of individual data}

The pooled OR for home paint exposure in the 1-3 months before conception using data from five studies was 1.54 (95\% CI 1.28, 1.85) (Table 3). In the analyses of immunophenotype, the increased risk was seen in B cell and not T cell ALL (ORs 1.52, 95\% CI $1.25,1.86$, and $1.0195 \%$ CI $0.60,1.67$ respectively) (Woolf's test for heterogeneity $p$ value 0.24 ). There was little difference when the analyses were stratified by child's sex, age at diagnosis, or year of birth (Table 3).

Using data from two studies, the pooled OR for home paint exposure in the year before conception and the risk of ALL was 1.00 (95\% CI 0.86, 1.17) (Table 3). There was little difference in the OR when the analyses were done by immunophenotype or when stratified by child's sex, or year of birth (Table 3 ). The OR was lower for those diagnosed before the age of 2 years, but this was based on small numbers.

The pooled OR for home paint exposure during pregnancy using eight studies analyses was 1.14 (95\% CI 1.04, 1.25) overall and was higher for B cell than T cell ALL (ORs 1.19, 95\% CI $1.08,1.31$, and $0.96,95 \%$ CI $0.75,1.21$ respectively) (Woolf's test for heterogeneity $p$ value 0.02 ) (Table 3 ). There was little difference when the analyses were stratified by child's sex, age or year of birth (Table 3 ) or by trimester of pregnancy ( $\sim 3000$ case and $\sim 4000$ controls from three studies, results not shown).

For those with exposure data for the 1-3 months before as well as during pregnancy, the ORs for the four-level exposure variable (exposure only before pregnancy, exposure only during pregnancy and exposure during both these time period, with no exposure before or during pregnancy as the reference group) were as follows; Only before pregnancy: 1.53 , 95\% CI 1.16, 2.03; only during pregnancy: OR 1.15, 95\% CI 1.02, 1.30; and in both time periods: $1.61,95 \%$ CI 1.26, 2.06 (Results not otherwise shown). The OR for exposure either in the 1-3 months before conception or during pregnancy was OR $1.25,95 \%$ CI 1.12, 1.39 (Results not otherwise shown). For those with exposure data for the year before as well as during pregnancy, however, the ORs were all similar (Only before pregnancy: 0.96, 95\% CI $0.78,1.19$; only during pregnancy: OR $1.05,95 \% \mathrm{CI} 0.82,1.35$; and in both time periods: $1.07,95 \%$ CI $0.87,1.30$ ) (Results not otherwise shown).

Using data from four studies, the pooled OR for exposure to paint around the home after birth was 1.22 (95\% CI 1.07, 1.39) (Table 3). When the analyses were restricted to the three studies that included exposures up until the reference date, the OR was 1.12 (95\% CI 0.94, 1.33) (Results not otherwise shown). There was little difference when the analyses were stratified by immunophenotype, child's sex, or year of birth (Table 3). The ORs for those diagnosed before the age of 2 years appeared to be higher than for other age groups (OR $1.53,95 \%$ CI $0.93,2.52$, age group interaction $p$ value 0.50 ), but there were fewer children in this age group. 
The ORs for either parent using paint in the year before pregnancy, or the mother using it during pregnancy were not elevated, while those for someone other than the parents doing the painting were elevated for all time periods (Table 3). The ORs for using oil-based paints were generally higher than for other paint types in both the year before pregnancy and during pregnancy, but were similar for painting after birth (Table 3).

There were sufficient studies and cases to do analyses by cytogenetic subtypes for paint exposure during pregnancy and after birth. For exposures during pregnancy, risk varied by cytogenetic subtype (Table 4); the ORs was highest among those with any 11q23/MLL rearrangement: 3.30 (95\% CI 1.71, 6.35). Most cases with any 11q23/MLL rearrangement were aged two years or under (66.6\%). The proportion of control mothers who reported paint exposure during pregnancy was inversely associated with the child's age, which suggested that parents of young children recalled more exposures than the parents of older children. Therefore, we restricted these analyses to subjects aged two years or younger. The resulting OR, based on 26 cases, was 2.60 (95\% 1.05, 6.43) (results not otherwise shown). Elevated ORs were also found in B cell cases with the presence of ETV6-Runx 1 and low hyperdiploidy (Table 4). The NCCLS contributed between $48-70 \%$ of cases for the cytogenetic subtype analyses. When the analyses were repeated excluding this study, the results were less precise, with an OR of $2.46(0.99,6.10)$ for any $11 \mathrm{q} 23 / M L L$ rearrangement and the other ORs were generally in the same direction and magnitude as when it was included (data not shown). For exposures after birth, only two studies had cytogenetic data and the NCCLS contributed $67-78 \%$ of the data. The ORs for B cell cases with low hyperdiploidy and those with the $t(12 ; 21)$ translocation were both elevated (Table 4). Excluding the NCCLS data, the OR among B cell cases with low hyperdiploidy was 1.57 (95\% CI 0.71, 3.48) while the OR for B cell cases with the $\mathrm{t}(12 ; 21)$ translocation was similar to that for all B cell cases from that study. There were insufficient cases to investigate $11 \mathrm{q} 23 / M L L$ rearrangements.

When the analyses for all time periods were repeated excluding children with Down syndrome (41 cases and four controls for the during pregnancy analyses and less for other time periods), there was little change in the results and there was also little difference when the analyses were adjusted for parents' occupational paint exposure or when the home and occupational exposure was combined into a single variable (data not shown).

\section{Discussion}

These pooled analyses add to the existing evidence that paint exposure around the home may be related to childhood ALL in certain circumstances. Using data from five studies, we found that paint exposure in the few months leading up to conception could be associated with an increased risk of ALL, and that this risk may be restricted to B cell ALL. By contrast, no association was found using data from the two studies with exposure data for the year before conception. Using data from eight studies, there was some evidence of association between paint exposure during pregnancy and an increased risk of B cell ALL, and among a subset of these studies, of certain cytogenetic subtypes (any 11q23/MLL rearrangement or ETV6-RunX-1 ( $\mathrm{t}(12 ; 21)$ translocations). Using data from four studies, there was evidence of a weak association between exposure after birth and ALL, which was 
more evident with certain cytogenetic subtypes and possibly in younger children. In addition, using data from a subset of studies, using any oil-based paints seemed to increase the risk of ALL before, during and after birth as did having someone other than the parents (likely to be a professional painter) paint the home. This last finding may reflect a higher dose or intensity of exposure, but we did not have the data to investigate this further. On the other hand, the risk associated with parents actively using the paint in relevant time periods appeared to be similar to any exposure in that time period.

Apart from the studies included in the pooled analyses which have previously publishing their findings $(6,4)$, there are only three other published reports $(12,7,5)$, that have investigated whether home paint exposure is associated with ALL. All were conducted in the US, and one included the subpopulation of ALL cases with Down syndrome (12), and thus its findings may have limited generalisability. While the study of Down Syndrome children found no association between exposure to paints, stains and lacquers in the month before or during pregnancy (12), Freedman et al (7) reported an OR of 1.2 (95\% CI 0.9, 1.5) for exposure to home painting in the year before birth, which is similar to our OR for the 1-3 months before or during pregnancy $(1.2,95 \%$ CI $1.1,1.4)$. The third previous non-CLIC study, based on 123 cases, investigated parental use of paints or lacquers during pregnancy or while the mother was breastfeeding and reported an increased risk with maternal paint use, but no association if the father or either parent used paint (5). The other potential source of paint exposure during pregnancy is maternal occupational paint exposure, but using a pooled sample of more than 8,000 cases from 12 CLIC studies, we had too few exposed mothers to draw any conclusions about maternal occupational exposure during pregnancy (3).

Only two studies other than the studies included in these pooled analyses have reported findings in relation to paint exposure after birth $(12,7)$. While the study restricted to children with Down Syndrome (12) found no association, the other (7), like ours found a weak association. In addition, they found that the risk was elevated with higher doses or frequency of exposure.

To the best of our knowledge, two of the studies in the current pooled analyses are the only previous reports by immunophenotype (6) or cytogenetic subtypes, $(6,4)$. Using this pooled sample, we found that the risk with paint exposure before birth was higher for B cell ALL than for T cell ALL, while ORs for exposure after birth were similar for both immunophenotypes; this could provide some insight into their different etiologies. It is plausible that prenatal exposures are more important for B cell ALL, which occurs in younger children. Not surprisingly, our pooled findings for exposure during pregnancy and after birth in relation to the most common cytogenetic subtype seen in childhood ALL, the ETV6-RunX-1 $\mathrm{t}(12 ; 21)$ translocation, are similar to the previously published data $(6,4)$ as these were the two studies in these analyses, and findings in both were in the same direction. However, with the larger sample size, our estimates are more precise. This translocation is thought to occur in utero as it has been detected in newborn blood samples (8), but a second postnatal event may be necessary to initiate disease (13). It is plausible that exposure to paints could be either the primary hit initiating DNA damage, or the subsequent event. The increased risk seen with exposure during pregnancy and 11q23/MLL rearrangement is novel. 
11q23/MLL rearrangement is predominantly seen in infant ALL and are thought to originate in utero during fetal hematopoiesis (14). Unlike other types of ALL, infant ALL is hypothesized to require only a single exposure in utero to trigger the disease (15). Among the two studies with 11q23/MLL rearrangement data, $66.7 \%$ of the cases were aged under two years, but they made up only about $\sim 13 \%$ of total cases in this age group from these studies. This may explain that while the OR $(1.29,95 \%$ CI $0.99,1.68)$ for children under two years was higher than for other age groups, it was not of the same magnitude as we found for $11 \mathrm{q} 23 / M L L$ rearrangement.

Our finding of an increased risk with painting close to the time of conception (in both the analyses of the individual time period and when combined with pregnancy) could support the hypothesis that environmental exposure results in paternal germ cell damage prior to fertilization. However, this is not supported by our previous finding that paternal occupational exposure to paints around conception was not associated with ALL risk (3) and one would assume that the frequency and level of exposure to paint chemicals would be much higher in occupational paint exposure than in home exposure. Perhaps the explanation for this is that the period when the painting was done may not reflect the true 'critical time of exposure'. For example, there is evidence that VOCs released by paints remain elevated in a home for at least a month after the painting occurred and that the levels were also high in rooms other than those painted (16). Therefore, levels of chemical residues could still be raised in the early weeks of pregnancy following painting done just before conception (or similarly levels could be raised after birth because of painting done in late pregnancy). Maternal exposure in early pregnancy could be critical, as hematopoiesis in the liver and bone marrow commences after the first month of fetal life, whereas in the extra-embryonic yolk sac it commences earlier (17).

Paints contain many individual compounds, some of which are thought to be carcinogenic (1). In addition, other potentially harmful agents can be associated with paint use, such as those used in preparation of surfaces or in the cleaning up process. About $75 \%$ of modern paints are water-based, while the remainder are oil-based and contain solvents such as toluene and xylene (18). Oil-based paints release VOCs into the atmosphere as do some water-based paints, albeit at lower levels (18). However, the composition of paints used over the relevant time periods for the studies would have changed, at least partly because of changing government legislation which continues to reduce VOC levels $(19,11)$. Using a subset of studies, we found that the OR associated with the use of any oil-based appeared to be higher than for only water-based paints, which suggests that compounds found in higher concentrations in oil-based paints could be implicated, but our findings are based on small numbers. However, if historically there was a risk with these paints, this risk may disappear with changing paint compositions and reducing VOC levels.

The major strength of this current investigation was the large sample size, especially for exposures during pregnancy to which all eight studies contributed data. While three of the studies (NCCLS (4), Australia, (6) and Canada (9)) included in the pooled analyses have previously published their findings in relation to paint exposure in the home, the other five studies had not. In addition, for these pooled analyses, we were able to include 50\% more NCCLS cases than were available for the previous report. The access to the original data 
allowed us to harmonize exposure data and other information such as immunophenotype. However, because the studies had collected data for different time periods before conception or after birth, or did not have these data at all, the different combinations of studies made it hard to judge whether changes to the OR reflected true differences by time period or were related to which studies were included, which is especially a concern for the two windows before pregnancy (1-3 months versus one year). Similarly, the interpretation of the analyses by cytogenetic subtypes is complicated as not all studies with data had information for all subtypes, thus changing the denominator. The pooled studies also included cases diagnosed over a fifteen year time period (1993-2008) so the availability and classification of the cytogenetic abnormalities of interest varied between studies. Therefore, we only included the classical subtypes the most routinely done in order to account at best for a part of the Bcell ALL cytogenetic heterogeneity while limiting the risk of inducing misclassifications due to insufficient data. Despite this, the analyses by cytogenetic subtypes lacked statistical power because of the limited number of cases.

Just as the definition of paint exposure varied across studies, so did the prevalence of exposure among the controls. The prevalence during pregnancy was $12-15 \%$ in the European studies with the definition of 'paint' or 'paint or varnish', $12 \%$ in the study using 'paints, lacquers, paint removers, turpentine products or thinners' (New Zealand), 19-30\% in the two US studies using 'paints, stains or lacquers' and 31-54\% in the two studies (Australia and Canada) with data on 'house painting'. It would be expected that studies which used a broad definition would have the highest prevalence as paint exposure from other sources, such as hobbies would have been included, but this was not the case. These differences may reflect true variations by region or when the studies were conducted. Other published estimates of the prevalence of paint exposure during pregnancy range from $19 \%$ controls who participated in a case-control study of fetal death in California (20), 44\% among controls in two of the previous case-control studies of paint and ALL in the US $(7,12)$ and $45 \%$ in the Danish National Birth Cohort $(21)$.

As our analyses used data derived from case-control studies, there is potential for recall bias. The individual studies attempted to minimize this by using standardized questionnaires. Nonetheless, this would not have removed the potential for case parents to think more deeply about past exposures and recall them more frequently (22). However, if this were the case, recall bias is unlikely to explain some of the findings, such as in relation to who did the painting (not the parents) or type of paint (only oil-based paint) or immunophenotype. Another explanation for some of our positive findings is chance.

In conclusion, these pooled analyses add to the existing evidence of a weak to modest association between painting in or around the home and the risk of childhood ALL, particularly B-cell ALL. We found that exposures close to conception, and, to a lesser extent those during pregnancy and in early childhood were associated with an increased risk in certain circumstances. The findings in relation to cytogenetic subtypes need to be replicated in a larger and more standardized sample. The existing evidence of cytogenetic and hematological changes in painters (1) adds weight to the plausibility of paint-induced DNA damage to the hematopoietic system at critical times of development being a precursor to childhood ALL. Until there is evidence to the contrary, we suggest that parents and those 
contemplating pregnancy limit paint use in the home in the year before birth and the child's early years.

\section{Supplementary Material}

Refer to Web version on PubMed Central for supplementary material.

\section{Acknowledgments}

\section{Funding}

The work reported in this paper by Helen Bailey was undertaken mostly during the tenure of a Postdoctoral Fellowship from the International Agency for Research on Cancer (IARC), partially supported by the European Commission FP7 Marie Curie Actions, - People- Co-funding of regional, national and international programmes (COFUND), with additional support by the Environment and Radiation Section of IARC. The CLIC administration, annual meetings, and pooled analyses are partially supported by the National Cancer Institute, NCI, USA (grant R03CA132172), National Institute of Environmental Health Sciences, NIEHS, USA (grants P01 ES018172 and R13 ES021145-01), the Environmental Protection Agency, EPA, USEPA, USA (grant RD83451101), the Children with Cancer, CwC, UK (Award No. 2010/097) and Alex's Lemonade Stand Foundation (grant 20140461).

Aus-ALL was supported by the Australian National Health and Medical Research Council (Grant ID 254539).

The Canadian study was funded by The National Cancer Institute of Canada; Grant numbers: \#014113, \#010735CERN \#RFA0405; The Medical Research Council of Canada; Grant number: MOP 37951; The Fonds de la recherche en santé du Québec; Grant number: \#981141; The Bureau of Chronic Disease Epidemiology, Canada; Health and Welfare Canada; The Leukemia Research Fund of Canada; and the National Health and Research Development Program, Ottawa.

France: ADELE Grant sponsors: INSERM, the French Ministère de l'Environnement, the Association pour la Recherche contre le Cancer, the Fondation de France, the Fondation Jeanne Liot, the Fondation WeisbremBerenson, the Ligue Contre le Cancer du Val de Marne, the Ligue Nationale Contre le Cancer.

NARECHEM, is supported in part by the National and Kapodistrian University, Athens, Greece.

The New Zealand Childhood Cancer Study was funded by the Health Research Council of NZ, the NZ Lottery Grants Board, the Otago Medical School (Faculty Bequest Funds), the Cancer Society of NZ, the Otago Medical Research Foundation, and the A.B. de Lautour Charitable Trust.

The Northern California Childhood Leukemia Study (NCCLS) is supported by the National Institutes of Health (NIH), USA (grants P01 ES018172, R01 ES09137, and P42-ES04705), Environmental Protection Agency (USEPA), USA (grant RD83451101), and the CHILDREN with CANCER (CwC), UK (former Children with Leukaemia) for data collection. The content is solely the responsibility of the authors and does not necessarily represent the official views of the NIH, USEPA, or the CwC.

COG: The E15 cohort of the Children's Oncology Group were funded by National Institutes of Health (NIH), USA (Grants R01CA049450 (E14) and R01CA048051 (E15)) and The Children's Cancer Research Fund, Minneapolis, $\mathrm{MN}$

We would like to thank Dr Maria S Pombo-de-Oliveira, Instituto Nacional de Câncer (INCA) who was one of experts in molecular biology who reviewed the consistency of CLIC cytogenetic data. We would like to thank our dear colleague and friend, Patricia Buffler, who passed away before the submission of this manuscript. She was a founding member and Chair of CLIC as well as the driving force behind the NCCLS. She provided unconditional support to finding the causes of childhood leukemia, and her scientific leadership and guiding forces within CLIC will be remembered.

The Aus-ALL consortium conducted the study and the Telethon Kids Institute (formerly Telethon Institute for Child Health Research; TICHR), University of Western Australia, was the coordinating centre. Bruce Armstrong (Sydney School of Public Health), Elizabeth Milne (TICHR), Frank van Bockxmeer (Royal Perth Hospital), Michelle Haber (Children's Cancer Institute Australia), Rodney Scott (University of Newcastle), John Attia (University of Newcastle), Murray Norris (Children's Cancer Institute Australia), Carol Bower (TICHR), Nicholas de Klerk (TICHR), Lin Fritschi (WA Institute for Medical Research, WAIMR), Ursula Kees (TICHR), Margaret Miller (Edith Cowan University), Judith Thompson (WA Cancer Registry) were the research investigators, and Helen Bailey (TICHR) was the project coordinator. The clinical Investigators were: Frank Alvaro (John Hunter Hospital, 
Newcastle); Catherine Cole (Princess Margaret Hospital for Children, Perth); Luciano Dalla Pozza (Children's Hospital at Westmead, Sydney); John Daubenton (Royal Hobart Hospital, Hobart); Peter Downie (Monash Medical Centre, Melbourne); Liane Lockwood, (Royal Children's Hospital, Brisbane); Maria Kirby (Women's and Children's Hospital, Adelaide); Glenn Marshall (Sydney Children's Hospital, Sydney); Elizabeth Smibert (Royal Children's Hospital, Melbourne); Ram Suppiah, (previously Mater Children's Hospital, Brisbane).

NARECHEM Greek Pediatric Hematology Oncology Clinicians: Margarita Baka MD, (Department of Pediatric Hematology -Oncology, "Pan.\&Agl. Kyriakou" Children's Hospital, Athens) Maria Moschovi MD, Sophia Polychronopoulou MD (Departments of Pediatric Hematology -Oncology, "A. Sophia” Children's Hospital, Athens); Emmanuel Hatzipantelis MD, PhD (Pediatric Hematology Oncology Unit, 2nd Pediatric Department of Aristotle University, AHEPA General Hospital, Thessaloniki); Maria Kourti MD (Pediatric Oncology Department, Hippokration Hospital, Thessaloniki); Eftychia Stiakaki MD (Department of Pediatric Hematology-Oncology, University Hospital of Heraklion, Heraklion); Ioannis Matsoukis MD (Department of Hygiene, Epidemiology and Medical Statistics, Athens University Medical School, 11527 Athens); Nick Dessypris, MSc, PhD and Evanthia Bouka, MPH: Department of Hygiene, Epidemiology and Medical Statistics, Athens University Medical School, 11527 Athens, Greece.

The New Zealand Childhood Cancer Study was co-ordinated at the University of Otago, where the study team included JD Dockerty, GP Herbison (who helped prepare data for this pooled analysis), DCG Skegg and JM Elwood. The names of the interviewers, secretaries, research assistants, clinicians, pathologists and cancer registry staff who contributed are listed in earlier publications from the NZ study.

COG: The E15 cohort of the Children's Oncology Group was identified by CCG (Children's Cancer Group) principle and affiliate member institutions. Further information can be found on the web-site: http:// www.curesearch.org/.

The NCCLS thanks the families for their participation and the clinical investigators at the following collaborating hospitals for help in recruiting patients: University of California Davis Medical Center (Dr. J. Ducore), University of California San Francisco (Drs. M. Loh and K. Matthay), Children's Hospital of Central California (Dr. V. Crouse), Lucile Packard Children's Hospital (Dr. G. Dahl), Children's Hospital Oakland (Dr. J. Feusner), Kaiser Permanente Roseville (former Sacramento; Drs. K. Jolly and V. Kiley), Kaiser Permanente Santa Clara (Drs. C. Russo, A. Wong, and D. Taggar), Kaiser Permanente San Francisco (Dr. K. Leung), and Kaiser Permanente Oakland (Drs. D. Kronish and S. Month). Finally, the NCCLS thanks the entire study staff and former University of California, Berkeley Survey Research Center for their effort and dedication.

The French authors would like to thank all of the Société Française de lutte contre les Cancers de l'Enfant et de l'Adolescent (SFCE) principal investigators: André Baruchel (Hôpital Saint-Louis/Hôpital Robert Debré, Paris), Claire Berger (Centre Hospitalier Universitaire, Saint-Etienne), Christophe Bergeron (Centre Léon Bérard, Lyon), Jean-Louis Bernard (Hôpital La Timone, Marseille), Yves Bertrand (Hôpital Debrousse, Lyon), Pierre Bordigoni (Centre Hospitalier Universitaire, Nancy), Patrick Boutard (Centre Hospitalier Régional Universitaire, Caen), Gérard Couillault (Hôpital d'Enfants, Dijon), Christophe Piguet (Centre Hospitalier Régional Universitaire, Limoges), Anne-Sophie Defachelles (Centre Oscar Lambret, Lille), François Demeocq (Hôpital Hôtel-Dieu, Clermont-Ferrand), Alain Fischer (Hôpital des Enfants Malades, Paris), Virginie Gandemer (Centre Hospitalier Universitaire - Hôpital Sud, Rennes), Dominique Valteau-Couanet (Institut Gustave Roussy, Villejuif), Jean-Pierre Lamagnere (Centre Gatien de Clocheville, Tours), Françoise Lapierre (Centre Hospitalier Universitaire Jean Bernard, Poitiers), Guy Leverger (Hôpital Armand-Trousseau, Paris), Patrick Lutz (Hôpital de Hautepierre, Strasbourg), Geneviève Margueritte (Hôpital Arnaud de Villeneuve, Montpellier), Françoise Mechinaud (Hôpital Mère et Enfants, Nantes), Gérard Michel (Hôpital La Timone, Marseille), Frédéric Millot (Centre Hospitalier Universitaire Jean Bernard, Poitiers), Martine Münzer (American Memorial Hospital, Reims), Brigitte Nelken (Hôpital Jeanne de Flandre, Lille), Hélène Pacquement (Institut Curie, Paris), Brigitte Pautard (Centre Hospitalier Universitaire, Amiens), Stéphane Ducassou (Hôpital Pellegrin Tripode, Bordeaux), Alain Pierre-Kahn (Hôpital Enfants Malades, Paris), Emmanuel Plouvier (Centre Hospitalier Régional, Besançon), Xavier Rialland (Centre Hospitalier Universitaire, Angers), Alain Robert (Hôpital des Enfants, Toulouse), Hervé Rubie (Hôpital des Enfants, Toulouse), Stéphanie Haouy (Hôpital Arnaud de Villeneuve, Montpellier), Christine Soler (Fondation Lenval, Nice), and Jean-Pierre Vannier (Hôpital Charles Nicolle, Rouen).

The Canada, Québec Study was conducted in the province over a twenty year period in all university-affiliated pediatric centers hospitals designated to diagnose and treat pediatric cancers, under the direction of Claire InfanteRivard. Main support collaborators were Alexandre Cusson, Marcelle Petitclerc and Denyse Hamer. We thank all families for their generous participation.

\section{Abbreviations}

ALL acute lymphoblastic leukemia 
Aus-ALL Australian Study of Causes of Acute Lymphoblastic Leukaemia in Children

CI Confidence interval

CLIC Childhood Leukemia International Consortium

COG Childhood Oncology Group (Children's Cancer Group)

NARECHEMNationwide Registration for Childhood Haemotological Malignancies

NCCLS Northern California Childhood Leukemia Study (USA)

NZCCS New Zealand Childhood Cancer Study.

OR Odds ratio

RDD random digit dialing

\section{References}

1. International Agency for Research on Cancer. Painting, Firefighting, and Shiftwork/ IARC Monographs in the evaluation of carcinogenic risks in humans vol 98. Lyon: International Agency for Research on Cancer; 2010.

2. Metayer C, Milne E, Clavel J, et al. The Childhood Leukemia International Consortium. Cancer Epidemiol. 2013; 37(3):336-347. [PubMed: 23403126]

3. Bailey HD, Fritschi L, Metayer C, et al. Parental occupational paint exposure and risk of childhood leukemia in the offspring: findings from the Childhood Leukemia International Consortium. Cancer Causes Control. 2014; 25(10):1351-1367. [PubMed: 25088805]

4. Scelo G, Metayer C, Zhang L, et al. Household exposure to paint and petroleum solvents, chromosomal translocations, and the risk of childhood leukemia. Environmental Health Perspectives. 2009; 117(1):133-139. [PubMed: 19165400]

5. Lowengart RA, Peters JM, Cicioni C, et al. Childhood leukemia and parents' occupational and home exposures. Journal of the National Cancer Institute. 1987; 79(1):39-46. [PubMed: 3474448]

6. Bailey HD, Milne E, de Klerk NH, et al. Exposure to house painting and the use of floor treatments and the risk of childhood acute lymphoblastic leukemia. International Journal of Cancer. 2011; 128(10):2405-2414. [PubMed: 20658537]

7. Freedman DM, Stewart P, Kleinerman RA, et al. Household solvent exposures and childhood acute lymphoblastic leukemia. American Journal of Public Health. 2001; 91(4):564-567. [PubMed: 11291366]

8. Wiemels JL, Cazzaniga G, Daniotti M, et al. Prenatal origin of acute lymphoblastic leukaemia in children. Lancet. 1999; 354(9189):1499-1503. [PubMed: 10551495]

9. Infante-Rivard C, Siemiatycki J, Lakhani R, et al. Maternal exposure to occupational solvents and childhood leukemia. Environmental Health Perspectives. 2005; 113(6):787-792. [PubMed: 15929905]

10. Tibbetts J. Under construction: building a safer industry. Environmental Health Perspectives. 2002; 110(3):A134-A141. [PubMed: 11882489]

11. Australian Paint Approval Scheme. Volatile Organic Compounds (VOC) Limits [internet]. 2006. http://wwwapasgovau/PDFs/D181pdf

12. Alderton LE, Spector LG, Blair CK, et al. Child and maternal household chemical exposure and the risk of acute leukemia in children with Down's syndrome: a report from the Children's Oncology Group. American Journal of Epidemiology. 2006; 164(3):212-221. [PubMed: 16760223]

13. Greaves M. Infection, immune responses and the aetiology of childhood leukaemia. Nature Reviews Cancer. 2006; 6(3):193-203. [PubMed: 16467884] 
14. Greaves MF, Wiemels J. Origins of chromosome translocations in childhood leukaemia. Nature Reviews Cancer. 2003; 3(9):639-649. [PubMed: 12951583]

15. Greaves MF, Maia AT, Wiemels JL, et al. Leukemia in twins: lessons in natural history. Blood. 2003; 102(7):2321-2333. [PubMed: 12791663]

16. Raw GJ, Coward SK, Brown VM, et al. Exposure to air pollutants in English homes. Journal of Exposure Analysis and Environmental Epidemiology. 2004; 14(Suppl 1):S85-S94. [PubMed: 15118750]

17. Tavian M, Biasch K, Sinka L, et al. Embryonic origin of human hematopoiesis. IntJ Dev Biol. 2010; 54(6-7):1061-1065. [PubMed: 20711983]

18. Van De Mark, MR., Sandefur, KD., Durham, KA. Kirk-Othmer Encyclopedia of Chemical Technology [Internet]. New York: Wiley InterScience. John Wiley \& Sons; 2005. Paint. p Available

19. European Commission. Report on the implementation of Directive 2004/42/EC of the European Parliament and of the Council on the limitation of emissions of volatile organic compounds due to the use of organic solvents in certain paints and varnishes and vehicle refinishing products and amending Directive 1999/13/EC. 2013 http://eur-lexeuropaeu/legal-content/EN/TXT/PDF/? uri=CELEX:52013DC0704\&rid=1.

20. Hertz-Picciotto I, Pastore LM, Beaumont JJ. Timing and patterns of exposures during pregnancy and their implications for study methods. Am J Epidemiol. 1996; 143(6):597-607. [PubMed: 8610677]

21. Sorensen M, Andersen AMN, Raaschou-Nielsen O. Non-occupational exposure to paint fumes during pregnancy and fetal growth in a general population. Environmental Research. 2010; 110(4): 383-387. [PubMed: 20219188]

22. Schuz J, Spector LG, Ross JA. Bias in studies of parental self-reported occupational exposure and childhood cancer. American Journal of Epidemiology. 2003; 158(7):710-716. [PubMed: 14507608] 


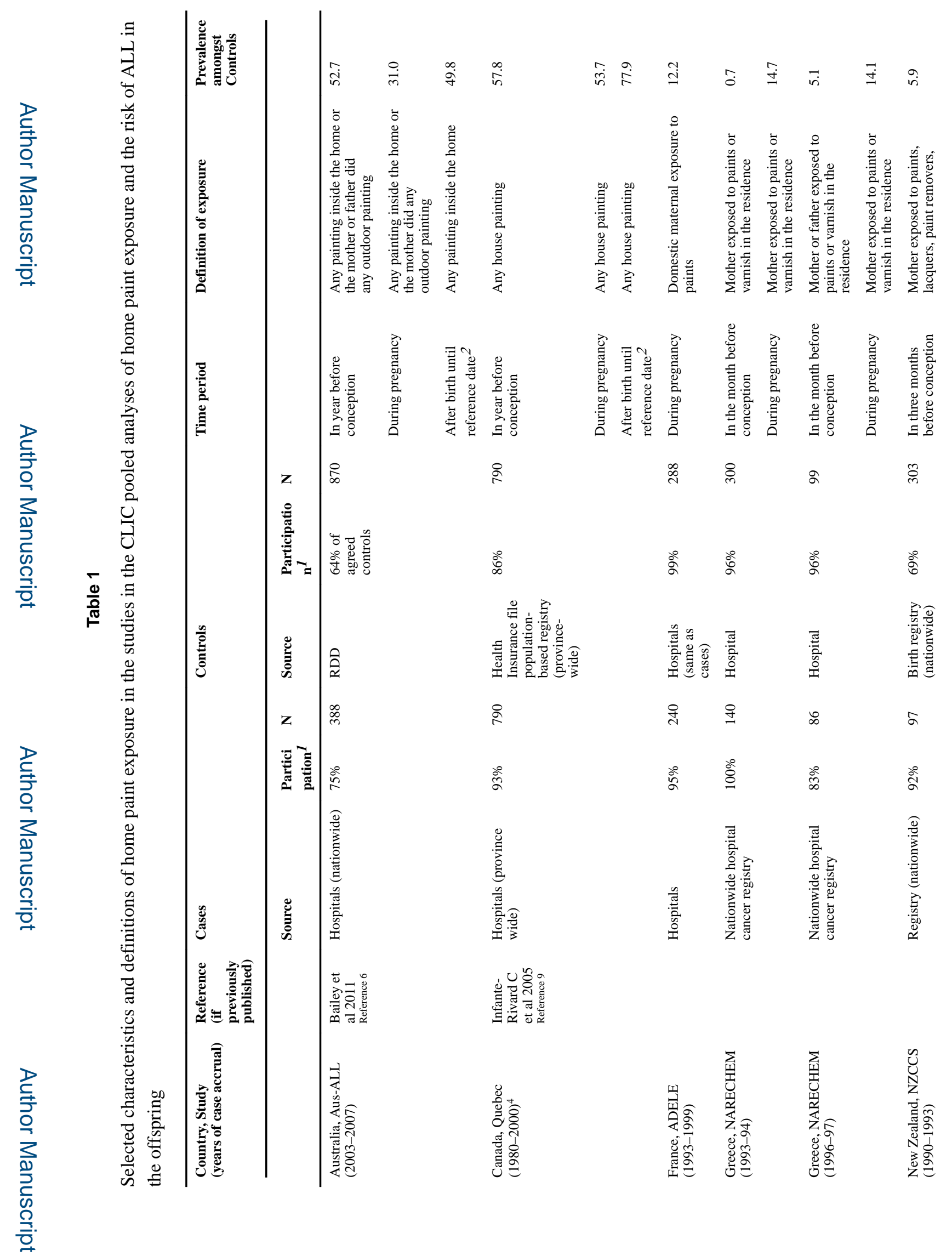

Cancer Causes Control. Author manuscript; available in PMC 2017 January 23. 
Bailey et al.

Page 16

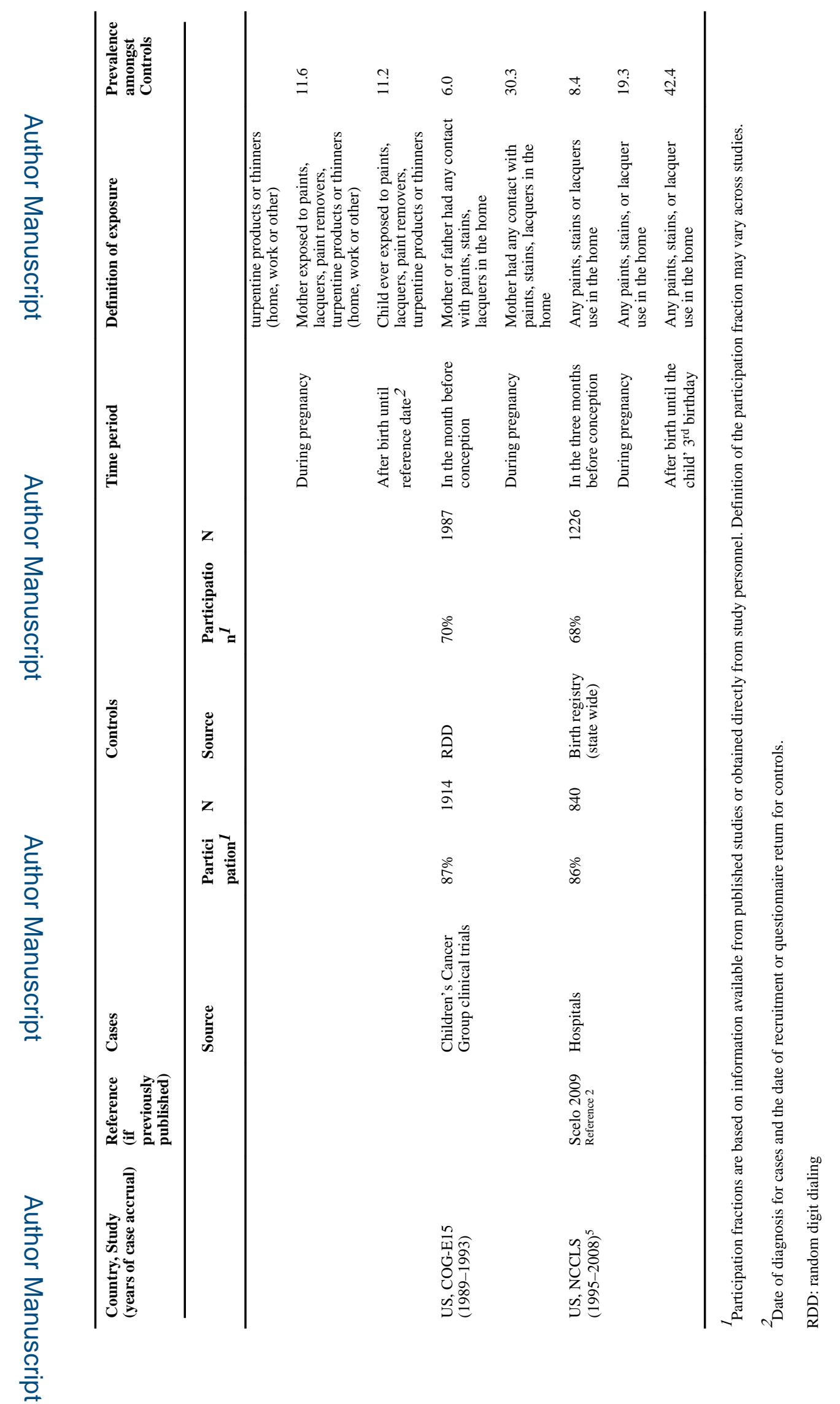

Cancer Causes Control. Author manuscript; available in PMC 2017 January 23. 


\section{Table 2}

Demographic and other characteristics of participants in the CLIC pooled analyses of home paint exposure and the risk of ALL in the offspring (8 studies)

\begin{tabular}{|c|c|c|c|c|}
\hline & \multicolumn{2}{|c|}{ Case $(n=4495)$} & \multicolumn{2}{|c|}{ Control $(n=5863)$} \\
\hline & $\mathbf{n}$ & $\% 1$ & $\mathbf{n}$ & $\% 1$ \\
\hline \multicolumn{5}{|l|}{ Type of ALL } \\
\hline B cell lineage & 3416 & 76.0 & & \\
\hline $\mathrm{T}$ cell lineage & 435 & 9.7 & & \\
\hline Other & 630 & 14.0 & & \\
\hline Missing & 14 & 0.3 & & \\
\hline \multicolumn{5}{|l|}{ Sex } \\
\hline Boy & 2513 & 55.9 & 3248 & 55.4 \\
\hline Girl & 1982 & 44.1 & 2615 & 44.6 \\
\hline \multicolumn{5}{|l|}{ Age (years) ${ }^{2}$} \\
\hline $0-1$ & 485 & 10.8 & 738 & 12.6 \\
\hline $2-4$ & 2096 & 46.6 & 2505 & 42.7 \\
\hline $5-9$ & 1319 & 29.3 & 1772 & 30.2 \\
\hline $10-14$ & 595 & 13.2 & 848 & 14.4 \\
\hline \multicolumn{5}{|l|}{ Year of birth } \\
\hline$<1985$ & 1116 & 24.8 & 1276 & 21.7 \\
\hline $1985-1988$ & 1258 & 28.0 & 1429 & 24.4 \\
\hline 1989-1995 & 1233 & 27.4 & 1594 & 27.2 \\
\hline 1996-2007 & 888 & 19.8 & 1564 & 26.7 \\
\hline \multicolumn{5}{|c|}{ Child's reference year ${ }^{3}$} \\
\hline 1980-1987 & 280 & 6.2 & 280 & 4.8 \\
\hline 1988-1993 & 2295 & 51.0 & 2541 & 43.3 \\
\hline 1994-2000 & 961 & 21.4 & 1333 & 22.7 \\
\hline 2001-2008 & 959 & 21.3 & 1709 & 29.1 \\
\hline \multicolumn{5}{|l|}{ Birth order } \\
\hline 1 st & 1954 & 43.5 & 2540 & 43.3 \\
\hline 2nd & 1571 & 34.9 & 2002 & 34.1 \\
\hline 3rd or more & 951 & 21.2 & 1295 & 22.1 \\
\hline Missing & 19 & 0.4 & 26 & 0.4 \\
\hline \multicolumn{5}{|c|}{ Mother's age at child's birth } \\
\hline$<25$ years & 1364 & 30.3 & 1584 & 27.0 \\
\hline $25-34$ years & 2633 & 58.6 & 3558 & 60.7 \\
\hline$>34$ years & 495 & 11.0 & 720 & 12.3 \\
\hline Missing & 3 & 0.1 & 1 & 0.0 \\
\hline \multicolumn{5}{|c|}{ Child has Down Syndrome } \\
\hline Yes & 41 & 0.9 & 4 & 0.1 \\
\hline No & 4452 & 99.0 & 5858 & 99.9 \\
\hline
\end{tabular}




\begin{tabular}{lcccc}
\hline & \multicolumn{2}{c}{ Case $(\mathbf{n}=\mathbf{4 4 9 5})$} & Control $(\mathbf{n}=\mathbf{5 8 6 3})$ \\
& $\mathbf{n}$ & $\mathbf{\%}$ & $\mathbf{n}$ & $\mathbf{\%}$ \\
\cline { 2 - 5 } & 2 & 0.0 & 1 & 0.0 \\
Missing & & & & \\
Highest level of education of either parent & & & & \\
Did not finish secondary education & 551 & 12.3 & 636 & 10.8 \\
Completed secondary education & 1895 & 42.2 & 2234 & 38.1 \\
Tertiary education & 2048 & 45.6 & 2989 & 51.0 \\
Missing & 1 & 0.0 & 4 & 0.1 \\
Ethnicity & & & & \\
White/Caucasian/European & 3432 & 76.4 & 4645 & 79.2 \\
Other & 1040 & 23.1 & 1180 & 20.1 \\
Indeterminate & 17 & 0.4 & 37 & 0.6 \\
Missing & 6 & 0.1 & 1 & 0.0 \\
\hline
\end{tabular}

${ }^{1}$ All percentages have been rounded to one decimal place and thus the totals may range from $99.9 \%-100.1 \%$

${ }^{2}$ Age groups are based on the child's age at the censoring date. For case, this was the date at diagnosis and for controls, it was the date that the study investigators nominated (either the date of recruitment or the date of the questionnaire return).

3 Reference years are based on the censoring date. For case, this was the date at diagnosis and for controls, it was the date that the study investigators nominated (either the date of recruitment or the date of the questionnaire return) 


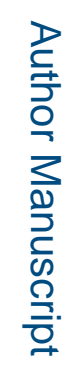

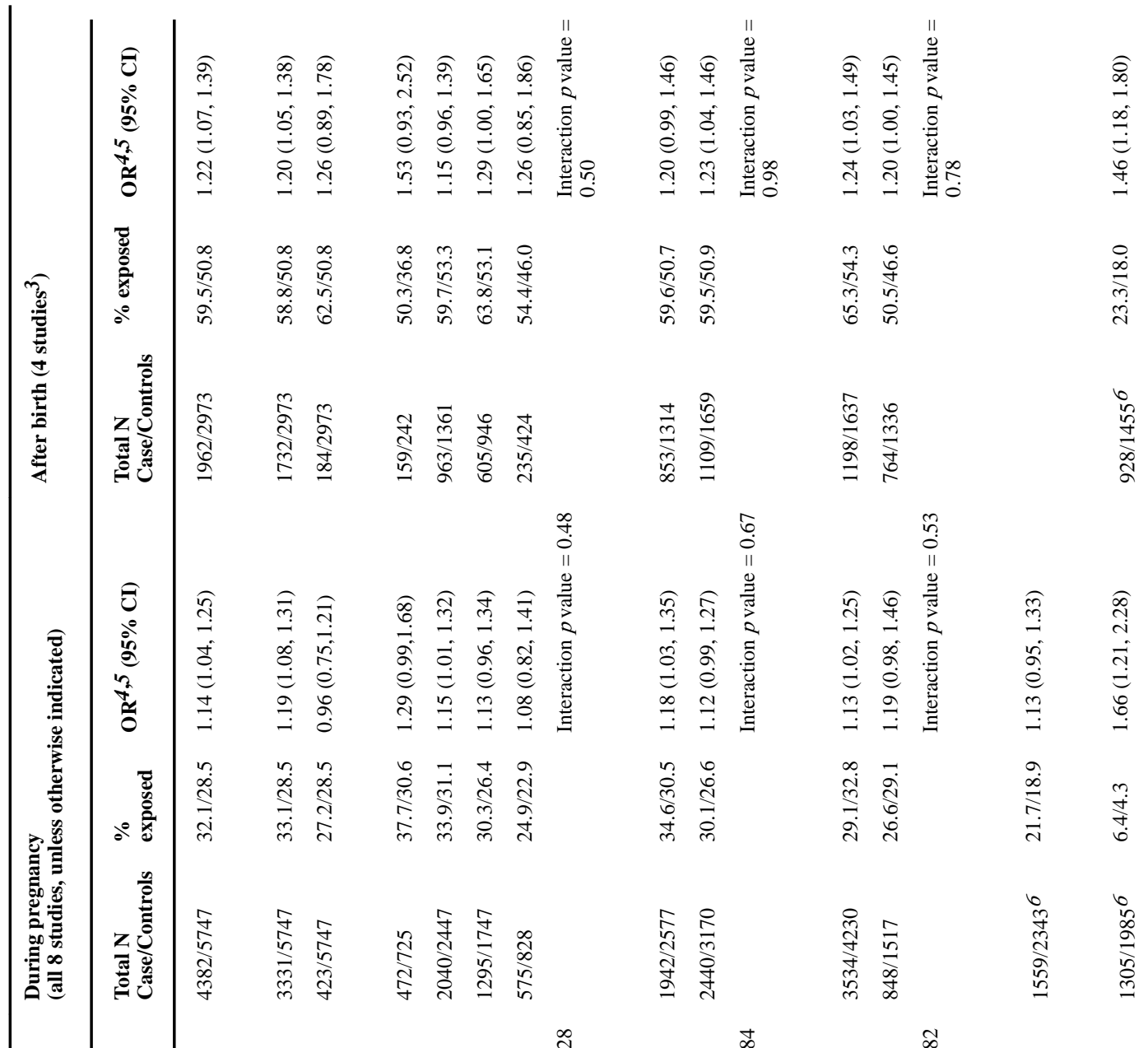

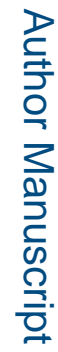

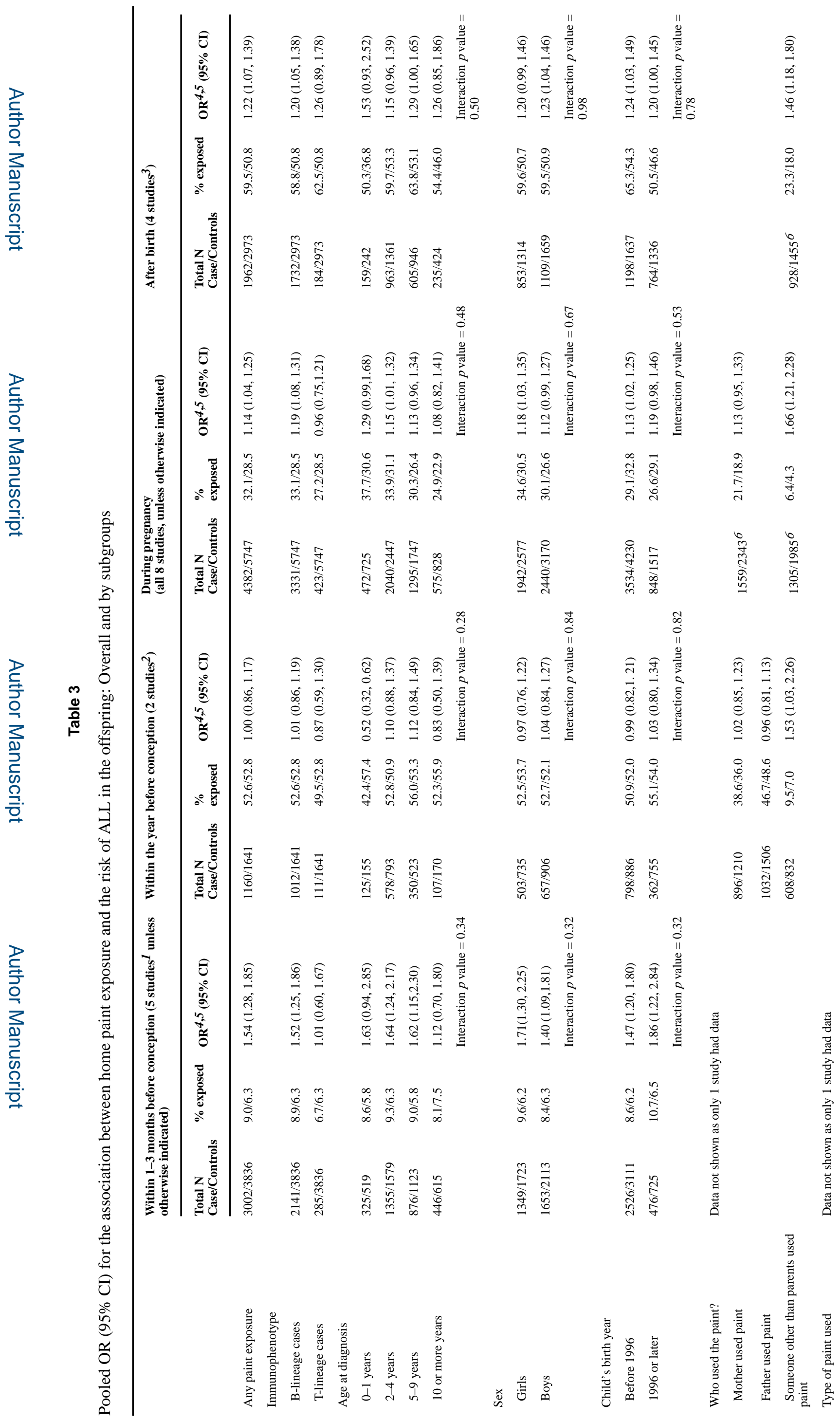




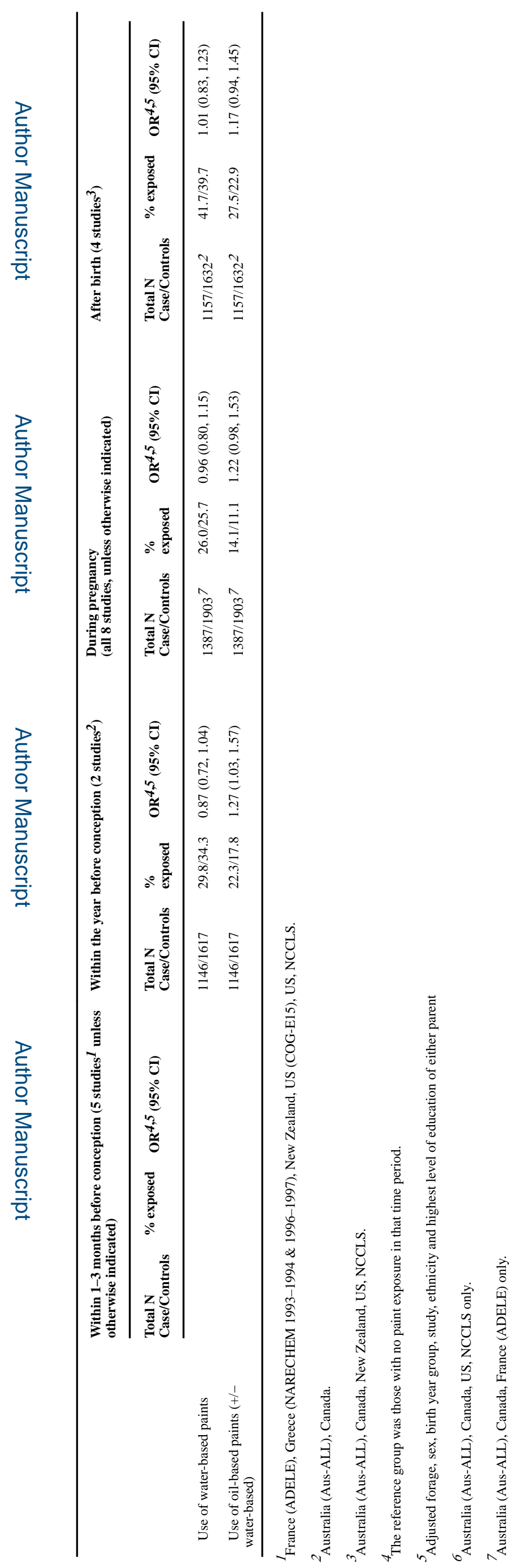




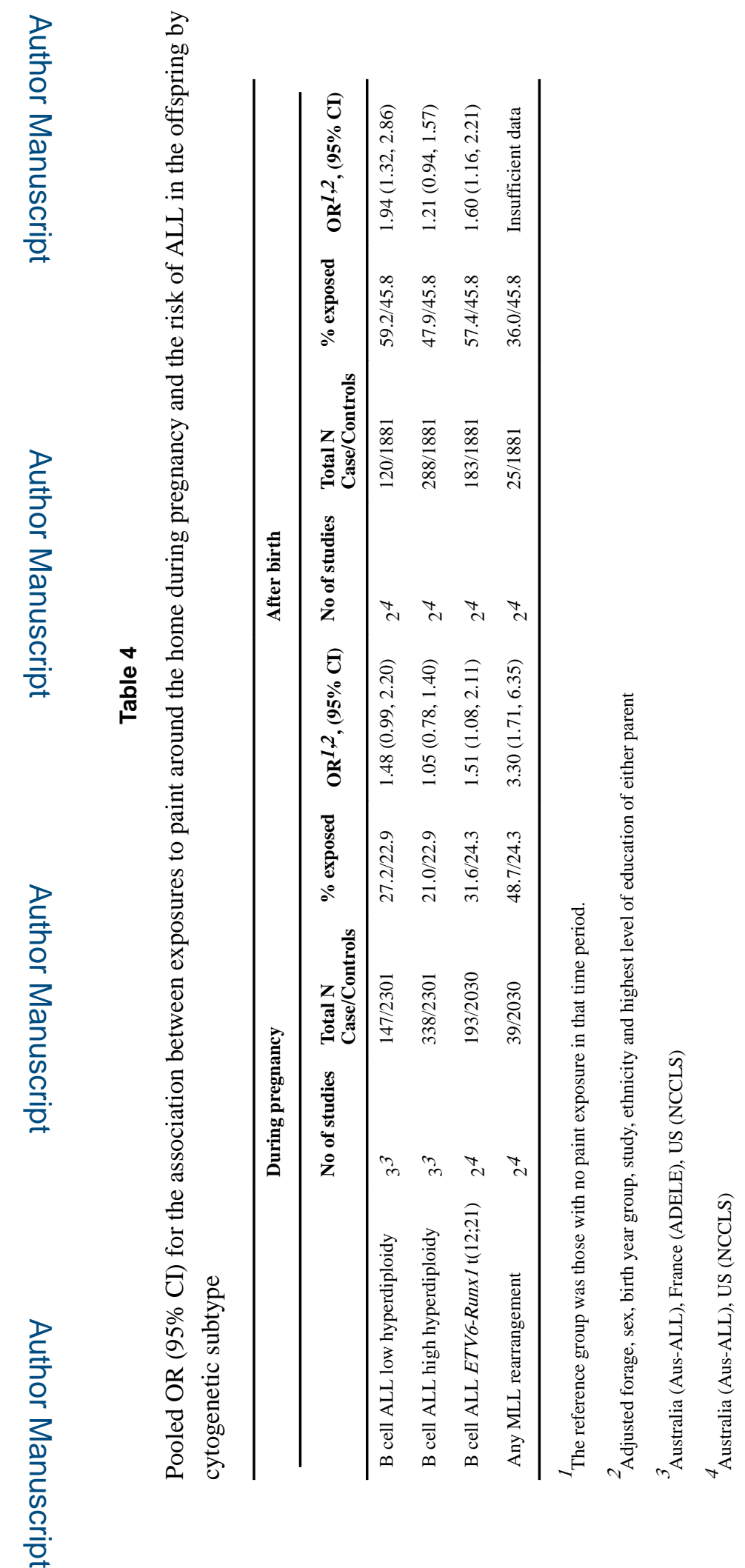

Cancer Causes Control. Author manuscript; available in PMC 2017 January 23. 